\title{
Twenty-two-year durability of Ionescu-Shiley pericardial aortic bioprosthesis implanted in a 49-year-old woman: A valuable insight into the performance of current pericardial bioprostheses
}

Charles A. Yankah, MD, PhD, Yuguo Weng, MD, Rudolf Meyer, MD, PhD, Henryk Siniawski, MD, PhD, and

Roland Hetzer, MD, PhD, Berlin, Germany

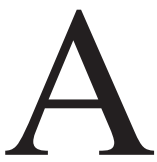

fter initial excellent hemodynamic results, the IonescuShiley pericardial bioprosthesis (Shiley, Inc, Irvine, Calif) demonstrated an unacceptably high rate of early structural deterioration. The valve was subsequently prohibited for clinical use in 1987 . We report the case of a patient with, to our knowledge, the longest durability of an Ionescu-Shiley pericardial bioprosthesis (ISPB), which is of historical value. This case provides some valuable insights into the performance of current pericardial bioprostheses.

\section{Clinical Summary}

In 1983 a 49-year-old woman underwent aortic valve replacement with a 25-mm ISPB and simultaneous replacement of the ascending aorta for type A dissection with a pre-existing aneurysm. In 2000 she underwent replacement of the aortic arch and descending aorta in combination with a prosthetic arterial bypass graft to the left subclavian artery. On the fifth postoperative day, reconstruction of the trachea was performed because of a tracheal injury. In 2003 the aneurysmal thoracoabdominal aorta was replaced with an aortic prosthesis at the level of the diaphragm, with an uneventful postoperative course.

Echocardiographic dimensions in 2000 were as follows: left ventricular (LV) end-diastolic diameter (LVEDD) $41 \mathrm{~mm}$; LV end-systolic diameter (LVESD) $30 \mathrm{~mm}$; LV posterior wall thickness in diastole/systole (D/S) 10/17 mm; interventricular septum in $\mathrm{D} / \mathrm{S}$ 10/11 mm; transvalvular mean gradient $6 \mathrm{~mm} \mathrm{Hg}$; LV ejection fraction $60 \%$; no aortic regurgitation.

Measurements in 2003 were as follows: LVEDD $52 \mathrm{~mm}$; LVESD $34 \mathrm{~mm}$; LV posterior wall thickness in D/S 11/17 mm; interventricular septum in D/S 13/16 mm; transvalvular mean gradient $10 \mathrm{~mm}$ $\mathrm{Hg}$; LV ejection fraction $60 \%$; no aortic regurgitation.

In December 2004 the patient presented with fever, and echocardiographic examination revealed prosthetic valve endocarditis with grade 2 aortic regurgitation. Abiotrophia adiacens, previously referred to as nutritionally variant streptococci, Streptococcus ad-

From Deutsches Herzzentrum, Berlin, Germany.

Received for publication March 9, 2006; accepted for publication April 10, 2006.

Address for reprints: Professor Charles A. Yankah, Department of Cardiovascular Surgery, German Heart Institute Berlin, Augustenburger Platz 1, Berlin 13353, Germany (E-mail: yankah@dhzb.de).

J Thorac Cardiovasc Surg 2006;132:427-8

$0022-5223 / \$ 32.00$

Copyright $@ 2006$ by The American Association for Thoracic Surgery doi:10.1016/j.jtcvs.2006.04.031

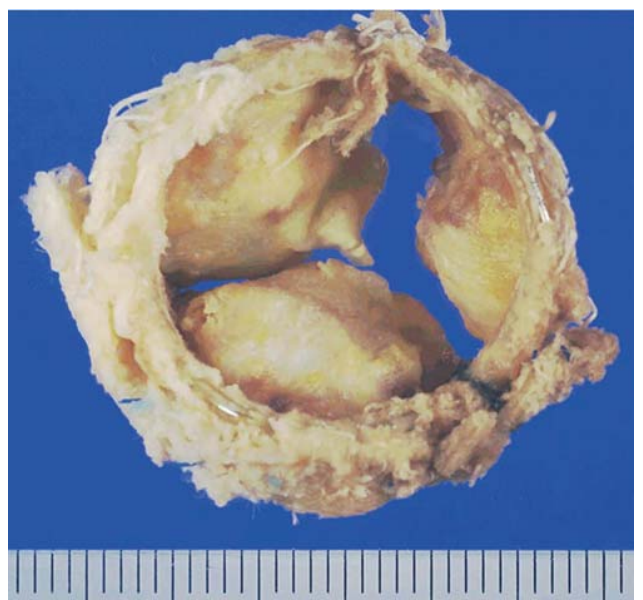

Figure 1. Explanted valve, ventricular view.

jacens, and Streptococcus defectivus, was identified in the blood cultures and was sensitive to ampicillin and gentamicin. She was discharged home after 14 days of hospitalization. On April 12,

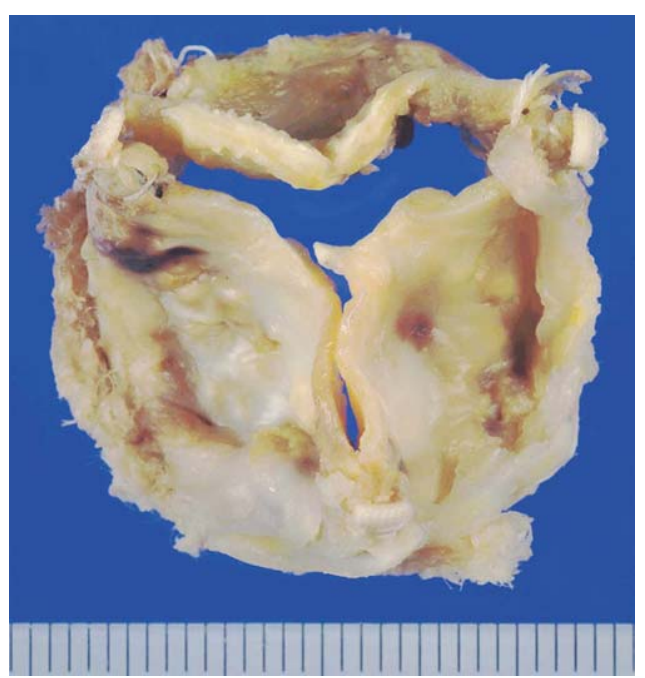

Figure 2. Explanted valve, aortic view. Retracted cusp with 2 remaining mobile cusps showing dystrophic changes with minor calcifications and irregularities at the coaptation surfaces. Multifocal scar tissue was found on the cusps without signs of infection. 
2005, she had increasing shortness of breath with intermittent chest pain, which led to rehospitalization for clinical assessment and treatment for heart failure. Echocardiographic study showed LVEDD $62 \mathrm{~mm}$, LVEF 45\%, and grade 3 aortic regurgitation of the ISPB. Blood chemistry studies showed no abnormalities. After successful treatment for heart failure and exclusion of coronary artery disease by coronary angiography, she underwent replacement of the ISPB with a 23-mm Hancock porcine bioprosthesis (Medtronic, Inc, Minneapolis, Minn) on April 25, 2005. The explanted ISPB showed dystrophic changes in all 3 cusps (Figures 1 and 2). The postoperative course was uneventful. The LV function had recovered after 3 months (ejection fraction 55\%, LVEDD $55 \mathrm{~mm}$ ). Clinically, she was in good cardiovascular condition (New York Heart Association functional class II) both at an outpatient visit in August 2005 and at a telephone interview on January 17, 2006.

\section{Comments}

This single case report demonstrates that there is potential in the overall performance of the new-generation pericardial bioprostheses, particularly in relation to structural valve deterioration in selected patients younger than 70 years of age.

We have learned from this single case that pericardial bioprosthesis in general can be implanted in a 50-year-old patient with comorbidities. Infective endocarditis of a pericardial prosthetic valve is a relatively uncommon complication occurring in $0.2 \%$ / patient-year, ${ }^{1}$ and its late occurrence is less common. Acceleration of structural deterioration of the pericardial tissue valve resulted in valve incompetence and compromised the LV function in this patient.

The low-profile ISPB demonstrated excellent hemodynamic performance and had preserved the LV function for more than 22 years. Finally, this case also supports the fact that new-generation pericardial bioprostheses may show the same long-term durability in patients younger than 70 years of age with comorbidities, as was demonstrated in our 17 -year clinical study, ${ }^{1}$ as well as in other publications. $^{2}$

We thank Professor Hans Borst for his contribution and Anne Gale for editorial assistance.

\title{
The disk of bileaflet mechanical mitral valve prosthesis jammed by ruptured papillary muscle
}

\author{
Marcin Fijalkowski, MD, Andrzej Koprowski, MD, PhD, Rafal Pawlaczyk, MD, PhD, Piotr Siondalski, MD, PhD, \\ Rafal Galaska, MD, Jan Rogowski, MD, PhD, and Andrzej Rynkiewicz, MD, PhD, Gdansk, Poland
}

$\mathrm{T}$ he incidence and natural history of papillary muscle rupture occurring after chord-sparing mitral valve replacement for ischemic mitral insufficiency are unknown. A case in which this complication occurred after chord-sparing mitral valve replacement is described.

\section{Clinical Summary}

A 59-year-old man was admitted to our institution with acute heart failure symptoms and chest discomfort lasting for 3 days. The

From the First Department of Cardiology and Department of Cardiosurgery, Medical University of Gdansk, Gdansk, Poland.

Received for publication April 10, 2006; accepted for publication April 20, 2006.

Address for reprints: Marcin Fijalkowski, MD, First Department of Cardiology and Department of Cardiosurgery, Medical University of Gdansk, ul. Debinki 7, 80-952 Gdansk, Poland (E-mail: mfijalkowski@amg.gda.pl).

J Thorac Cardiovasc Surg 2006;132:428-30

$0022-5223 / \$ 32.00$

Copyright $\odot 2006$ by The American Association for Thoracic Surgery doi:10.1016/j.jtcvs.2006.04.018

\section{References}

1. Yankah AC, Schubel J, Buz S, Siniawski H, Hetzer R. Seventeen-year clinical results of 1,037 Mitroflow pericardial heart valve prostheses in the aortic position. J Heart Valve Dis. 2005;14:172-9.

2. Minami K, Boethig D, Mirow N, Kleikamp G, Koertke H, Godehardt E, et al. Mitroflow pericardial valve prosthesis in the aortic position: an analysis of long-term outcome and prognostic factors. J Heart Valve Dis. 2000;9:112-22. electrocardiogram showed sinus tachycardia (115 beats/min) and pathologic Q waves in the II, III, and aVF leads. The serum concentrations of creatine kinase $\mathrm{MB}$ and troponin I were significantly elevated. A chest radiograph revealed pulmonary edema. Two-dimensional transthoracic echocardiography showed akinesia of inferior and posterior walls and severe mitral regurgitation caused by posterior leaflet restriction (vena contracta $7 \mathrm{~mm}$ ). Coronary angiography showed occluded right and circumflex coronary arteries and a critically narrowed left anterior descending coronary artery.

On the basis of these findings, mitral valve surgery and coronary artery bypass grafting were recommended. Treatment with intra-aortic balloon pump support was started, without significant improvement. Emergency cardiosurgery was performed because of the patient's unstable condition. The mitral valve was replaced with a 29-mm Medtronic Advantage prosthesis (Medtronic, Inc, Minneapolis, Minn) placed in antianatomic position with preservation of the posterior leaflet (ring annuloplasty was not successful). Coronary artery revascularization was done in the same operation.

The early postoperative course was uneventful. At the end of the second week of hospitalization, hypotension and pulmonary 